\title{
Hyperpolarizing GABAergic Transmission Requires the KCC2 C-Terminal ISO Domain
}

\author{
Brooke A. Acton, ${ }^{1}$ Vivek Mahadevan, ${ }^{1}$ Adrianna Mercado ${ }^{2,3}$ Pavel Uvarov,${ }^{4}$ Yanli Ding, ${ }^{2}$ Jessica Pressey, ${ }^{1}$ \\ Matti S. Airaksinen, ${ }^{4}$ David B. Mount, ${ }^{2,5}$ and Melanie A. Woodin ${ }^{1}$ \\ ${ }^{1}$ Department of Cell and Systems Biology, University of Toronto, Toronto, Ontario, Canada M5S 3G5, ${ }^{2}$ Renal Division, Brigham and Women's Hospital, \\ Harvard Medical School, Boston, Massachusetts 02115, ${ }^{3}$ Departamento de Nefrología, Instituto Nacional de Cardiología Ignacio Chávez, 14080 Mexico City, \\ Mexico, ${ }^{4}$ Neuroscience Center and Institute of Biomedicine, University of Helsinki, FIN-00014 Helsinki, Finland, and ${ }^{5}$ Renal Division, Veterans Affairs \\ Boston Healthcare System, Harvard Medical School, West Roxbury, Massachusetts 02132
}

$\mathrm{KCC} 2$ is the neuron-specific member of the of $\mathrm{K}^{+}-\mathrm{Cl}^{-}$cotransporter gene family. It is also the only member of its family that is active under physiologically normal conditions, in the absence of osmotic stress. By extruding $\mathrm{Cl}^{-}$from the neuron under isotonic conditions, this transporter maintains a low concentration of neuronal $\mathrm{Cl}^{-}$, which is essential for fast inhibitory synaptic transmission by $\mathrm{GABA}$ and glycine in the mature nervous system. The other members of this $\mathrm{K}^{+}-\mathrm{Cl}^{-}$cotransporter gene family are exclusively swelling-activated. Here we demonstrate that a 15 aa region near the end of the $\mathrm{C}$ terminus, unique to KCC2 (termed the ISO domain), is required for KCC2 to cotransport $\mathrm{K}^{+}$and $\mathrm{Cl}^{-}$out of the neuron under isotonic conditions. We made this discovery by overexpressing chimeric $\mathrm{KCC} 2-\mathrm{KCC} 4$ cDNA constructs in cultured hippocampal neurons prepared from Sprague Dawley rat embryos and assaying neuronal $\mathrm{Cl}^{-}$through gramicidin perforated patch-clamp recordings. We found that when neurons had been transfected with a chimeric KCC2 that lacked the unique ISO domain, hyperpolarizing responses to GABA were abolished. This finding indicates that the ISO domain is required for neuronal $\mathrm{Cl}^{-}$regulation. Furthermore, we discovered that when KCC2 lacks the ISO domain, it still retains swelling-activated transport, which demonstrates that there are exclusive molecular determinants of isotonic and swelling-induced $\mathrm{K}^{+}-\mathrm{Cl}^{-}$cotransport in neurons.

\section{Introduction}

KCC2 is a member of the Slc12a gene family of $\mathrm{K}^{+}{ }_{-} \mathrm{Cl}^{-}$cotransporters. This family of cotransporters was first identified for their volume-regulatory properties in nonneuronal cells (Lauf and Bauer, 1987; Payne et al., 1996). However, KCC2 is now most widely recognized for its ability to extrude $\mathrm{Cl}^{-}$from neurons (Rivera et al., 1999; Blaesse et al., 2009). Low neuronal $\mathrm{Cl}^{-}$is required for $\mathrm{GABA}_{\mathrm{A}}$ inhibitory synaptic transmission in the CNS. KCC2 is unique compared with its family members for two primary reasons: first, because it is expressed exclusively in neurons (Payne et al., 1996; Rivera et al., 1999; Strange et al., 2000); and second, because it is the only transporter that can extrude $\mathrm{Cl}^{-}$ under isotonic conditions (Payne et al., 1996; Strange et al., 2000).

KCC2 is essential for proper nervous system function, as decreased KCC2 expression results in neuropathic pain, spasticity

Received Dec. 8, 2011; revised May 10, 2012; accepted May 11, 2012.

Author contributions: B.A.A., A.M., P.U., M.S.A., D.B.M., and M.A.W. designed research; B.A.A., V.M., A.M., P.U., Y.D., J.P., D.B.M., and M.A.W. performed research; B.A.A., V.M., and M.A.W. analyzed data; B.A.A. and M.A.W. wrote the paper.

This work was supported by grants from Natural Sciences and Engineering Research Council to M.A.W. and grants from the National Institutes of Health to D.B.M. (DK57708 and DK070756) and from the Academy of Finland to M.S.A. We thank Lawrence Kwok, Jonah Chevrier, and Michelle Huang for their experimental assistance.

Correspondence should be addressed to either of the following Melanie A. Woodin, 25 Harbord Street, Toronto, Ontario, Canada M5S 3G5, E-mail:m.woodin@utoronto.ca; or David B. Mount, Brigham and Woman's Hospital Room 542, Harvard Institutes of Medicine, 4 Blackfan Circle, Boston, MA 02115. E-mail: dmount@rics.bwh.harvard.edu.

DOI:10.1523/JNEUROSCI.6089-11.2012

Copyright $\odot 2012$ the authors $\quad 0270-6474 / 12 / 328746-06 \$ 15.00 / 0$ following spinal cord injury, epileptic seizures, and death at birth due to respiratory failure (Kahle et al., 2008; Blaesse et al., 2009). Moreover, KCC2 is the key protein underlying spike-timingdependent plasticity of GABAergic synapses (Woodin et al., 2003; Ormond and Woodin, 2009).

While all $\mathrm{K}^{+}-\mathrm{Cl}^{-}$cotransporters are activated by cell swelling (Lauf and Bauer, 1987; Flatman, 2008), KCC2 is the only family member that is also able to extrude $\mathrm{Cl}^{-}$in the absence of osmotic stress. KCC2 functions under isotonic conditions by deriving energy from the $\mathrm{K}^{+}$gradient established by the $\mathrm{Na}^{+}-\mathrm{K}^{+}$-ATPase to extrude $\mathrm{Cl}^{-}$(Payne et al., 1996). As a result, KCC2 maintains a low concentration of intracellular $\mathrm{Cl}^{-}\left(\left[\mathrm{Cl}^{-}\right]_{\mathrm{i}}\right)$, which upon $\mathrm{GABA}_{\mathrm{A}} \mathrm{R}$ activation allows $\mathrm{Cl}^{-}$influx down its electrochemical gradient. The failure thus far to define the molecular determinant(s) of constitutive $\mathrm{K}^{+}-\mathrm{Cl}^{-}$cotransport mediated by KCC2 represents a large gap in our fundamental knowledge regarding neuronal $\mathrm{Cl}^{-}$regulation and inhibitory synaptic transmission.

To determine what confers KCC2 with this unique ability to transport $\mathrm{Cl}^{-}$isotonically, we took a gene modification approach and assayed neuronal $\mathrm{Cl}^{-}$regulation. Based on our previous work using a $\mathrm{Rb}^{+}$-uptake assay that demonstrated that a C-terminal domain renders KCC2 constitutively active in Xenopus oocytes (Mercado et al., 2006), we asked whether this same domain also confers KCC2 with the ability to extrude $\mathrm{Cl}^{-}$from neurons under physiologically relevant isotonic conditions. Our findings provide the first demonstration of a KCC2-specific C-terminal domain responsible for conferring constitutively active isotonic transport in neurons. Moreover, we discovered that 


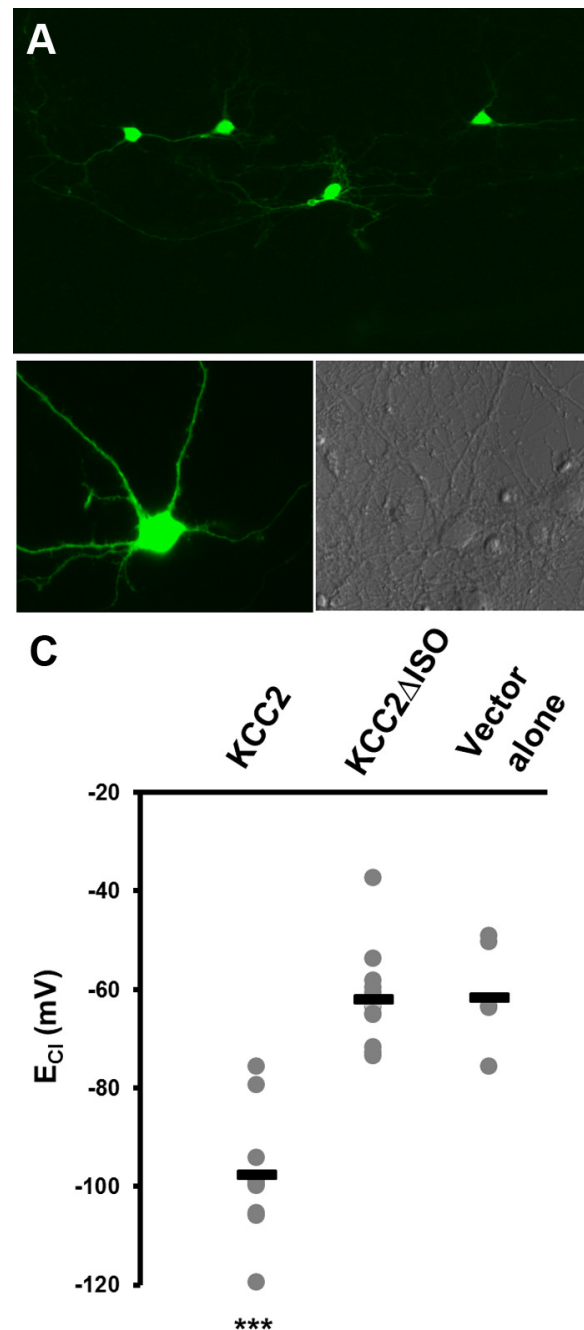

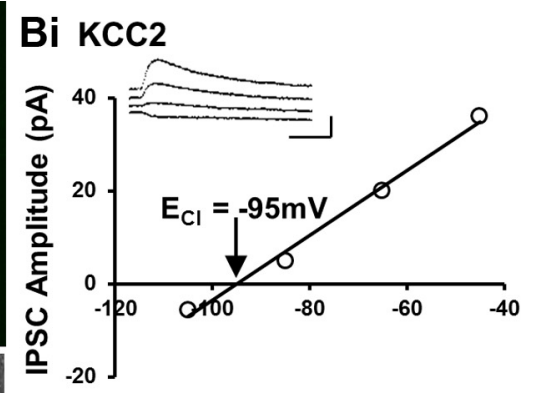

$\mathrm{Bii} \mathrm{KCC2 \triangle ISO}$

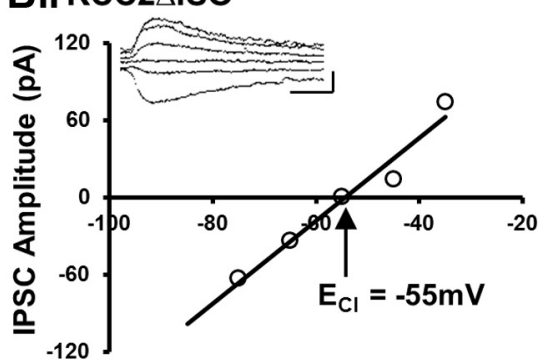

Biii Vector alone

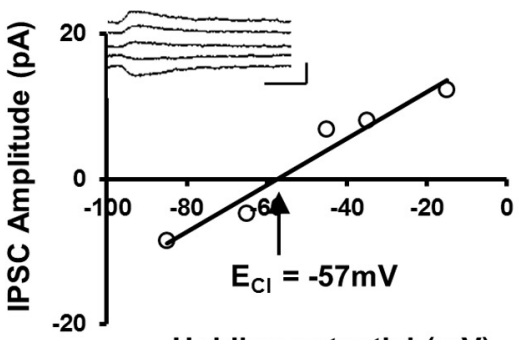

Holding potential $(\mathrm{mV})$

Figure 1. $\mathrm{KCC} 2 \Delta \mathrm{ISO}$ does not transport $\mathrm{K}^{+}$or $\mathrm{Cl}^{-}$under isotonic conditions. $\boldsymbol{A}$, Fluorescent images of cells overexpressing $\mathrm{KCC} 2 \Delta \mathrm{ISO}$; transfected neurons were chosen for electrophysiological experiments based on their expression of GFP. $\boldsymbol{B}$, Examples of gramicidin perforated patch-clamp recordings obtained from neurons overexpressing $\mathrm{KCC}(\boldsymbol{i}), \mathrm{KCC} \triangle \mathrm{ISO}$ (ii), and vector alone (iii). The IPSC amplitude was plotted against the holding potential, and the intercept of this curve with the $x$-axis was taken as $E_{C I}$ (arrows). Insets, Traces of IPSCs for the examples shown. Scale bars: $60 \mathrm{pA}, 150 \mathrm{~ms}$. C, Summary of all experiments similar to $\boldsymbol{B}$ shown as a distribution plot. ${ }^{* * *} p<0.001$.

this KCC2-unique domain is not required for the cell swellinginduced activation of KCC2 in neurons, demonstrating that separate molecular mechanisms determine KCC2 function during physiological and presumably pathophysiological states.

\section{Materials and Methods}

Hippocampal culture. Low-density cultures of dissociated rat hippocampal neurons were prepared as previously described from E18 Sprague Dawley rats (Balena et al., 2010). Briefly, E18 embryos of either sex were decapitated in accordance to guidelines from the University of Toronto Animal Care Committee and the Canadian Council on Animal Care. Hippocampi were then removed and treated with trypsin for 15 min at $37^{\circ} \mathrm{C}$, followed by gentle trituration. The dissociated cells were plated at an approximate density of $50,000 \mathrm{cells} / \mathrm{ml}$ on poly-D-lysinecoated 25 and $10 \mathrm{~mm}$ glass coverslips, and placed in $35 \mathrm{~mm}$ Petri dishes. Cells were plated in Neurobasal medium (Invitrogen), supplemented with 2\% B-27 (Invitrogen) and 10\% FBS (Sigma-Aldrich). Twenty-four hours after plating, one-third of the medium was replaced with original plating medium, and then changed again every $7 \mathrm{~d}$. Recordings were made after $10-13 \mathrm{~d}$ in culture (DIC). Lipofectamine 2000 was used to transfect cultured neurons with chimeric cDNA constructs.
DNA constructs. The KCC2 $2 \mathrm{ISO}$ construct was generated by serial mutagenesis of hKCC2 (QuikChangeII; Stratagene), replacing residues 1022-1037 with the equivalent region of KCC4; the entire open reading frame was sequenced to verify the successful final sequence and exclude PCR-generated errors. The other cDNA constructs were the same as those we generated previously (Mercado et al., 2006). Briefly, all of the constructs are composed of the cDNA of the gene of interest in the bicistronic pMES vector (gift from Karl Kandler, University of Pittsburgh, Pittsburgh, PA) (Lee et al., 2005), which contains a cytomegalovirus enhancer, a $\beta$-actin promoter, internal ribosome entry site, and an EGFP reporter. In addition, we generated extracellular-tagged KCC2-HA and KCC2 $\Delta$ ISO-HA constructs to quantify their membrane expression. Briefly, a triple tandem copy of the influenza virus hemagglutin (HA) peptide (YPYDVPDYA) was inserted into the second predicted extracellular loop of hKCC2 and KCC2 2 ISO proteins. For this purpose, an $\sim 700 \mathrm{bp}$ fragment of human KCC2 cDNA sequence between unique MunI and SacI restriction sites was synthesized using the BlueHeron Gene Synthesis Service (Blue Heron Biotech). This fragment contained a sequence encoding a triple tandem copy of the HA peptide inserted into exactly the same position as has been shown previously to preserve mouse KCC2 protein function and membrane expression (Zhao et al., 2008).

Electrophysiology. Perforated patch-clamp recordings were performed using pipettes made from glass capillaries (WPI). For perforated recordings, pipettes with a resistance of 7-12 $\mathrm{M} \Omega$ were filled with an internal solution containing $150 \mathrm{~mm} \mathrm{KCl,} 10 \mathrm{~mm}$ HEPES, and 50 $\mu \mathrm{g} / \mathrm{ml}$ gramicidin ( $\mathrm{pH} 7.4,300 \mathrm{mOsm}$ ). For $\mathrm{Cl}^{-}$loading experiments, pipettes (5-7 $\mathrm{M} \Omega$ ) were filled with an internal solution containing the following (in $\mathrm{mm}$ ): $90 \mathrm{~K}^{+}$-gluconate, 50 $\mathrm{KCl}, 10$ HEPES, 0.2 EGTA, 4 ATP, 0.3 GTP, and 10 phosphocreatine ( $\mathrm{pH} 7.4,300 \mathrm{mOsm})$. Neurons were perfused $(\sim 1 \mathrm{ml} / \mathrm{min})$ with either isotonic or hypotonic extracellular solution [isotonic (in mM): $150 \mathrm{NaCl}, 3 \mathrm{KCl}, 3 \mathrm{CaCl} 2 \cdot 2 \mathrm{H} 2 \mathrm{O}, 2 \mathrm{MgCl} 2 \cdot 6 \mathrm{H} 2 \mathrm{O}$, 10 HEPES, 5 glucose at $\mathrm{pH}$ 7.4, 301-315 mOsm; hypotonic: (in mM) 75 $\mathrm{NaCl}, 1.5 \mathrm{KCl}, 1.5 \mathrm{CaCl} 2 \cdot 2 \mathrm{H} 2 \mathrm{O}, 2 \mathrm{MgCl} 2 \cdot 6 \mathrm{H} 2 \mathrm{O}, 5 \mathrm{HEPES}, 2.5$ glucose at pH 7.4, 180-185 mOsm). Recordings were performed with a MultiClamp 700B (Molecular Devices) patch-clamp amplifier. Signals were filtered at $5 \mathrm{kHz}$ using amplifier circuitry. Data were acquired and analyzed using Clampfit 9 (Molecular Devices). Recordings started when the series resistance dropped below $50 \mathrm{M} \Omega$. Current-voltage measurements were made by $30 \mathrm{~s}$ steps depolarizations from -85 to $-35 \mathrm{mV}$ in voltageclamp mode, during which a $10 \mu \mathrm{M}$ GABA puff was applied to the soma using a picospritzer. A linear regression of the IPSC amplitude was used to calculate the voltage dependence of IPSCs; the intercept of this line with the abscissa was taken as $E_{\mathrm{Cl}}$ (experiments were performed in bicarbonate-free solution buffered with HEPES, the $\mathrm{GABA}_{\mathrm{A}}$ current was solely mediated by $\mathrm{Cl}^{-}$, and thus we report $E_{\mathrm{Cl}}$ and not $E_{\mathrm{GABA}}$ ). Electrophysiological values have not been corrected for the liquid junction potential of $\sim 7 \mathrm{mV}$. The expected $\left[\mathrm{Cl}^{-}\right]_{\mathrm{i}}$ was calculated using the Nernst equation.

Live immunostaining. Coverslips containing the cultured neurons were washed for $5 \mathrm{~min}$ at $37^{\circ} \mathrm{C}$ in isotonic extracellular solution (XCS). The coverslips were incubated in mouse monoclonal anti-HA antibody (HA.11; Covance) at $37^{\circ} \mathrm{C}$ for $1 \mathrm{~h}$. Cells were washed once for $5 \mathrm{~min}$ in 
isotonic XCS and then incubated with the secondary Alexa Fluor 555 goat anti-mouse antibody (Invitrogen) for $1 \mathrm{~h}$ in the dark at $37^{\circ} \mathrm{C}$. The coverslips were imaged while being perfused with isotonic XCS at room temperature to mimic electrophysiological recording conditions.

Confocal microscopy and image analysis. Immunofluorescent images were acquired on a Wave FX spinning disc confocal system (Quorum Technologies) with Zeiss Observer $\mathrm{Z1}$ inverted microscope, using a $43 \times 1.4$-NA oilimmersion objective. Z-stacks were acquired at a pixel resolution of $1024 x y$ with a $z$-step of 0.2 $\mu \mathrm{m}$. Samples were acquired sequentially using line-lambda mode at excitation wavelengths of $488 \mathrm{~nm}$ (for GFP) and $568 \mathrm{~nm}$ (for Cy3). Images were acquired and analyzed using Volocity 3D Image Analysis software (PerkinElmer). A minimum of 12 cells were imaged and analyzed from four coverslips in each condition. We measured anti-HA (Cy3) immunofluorescence cluster intensity in the $z$-plane, under constant illumination. The soma and the first $50 \mu \mathrm{M}$ of the most prominent primary dendrite of GFP-positive neurons were selected as the area of interest (AOI). Clusters were defined as regions within the AOI whose fluorescence in-

tensity was significantly greater than that of the average fluorescence intensity within the field of view. The anti-HA intensity for KCC2 $\Delta \mathrm{ISO}$ was calculated as a percentage of anti-HA intensity of KCC2-HA after background subtraction.

Statistics. Results are given as mean \pm SEM. For the results in Figures $1 C, 2 B, 3 B$, and $4 A$, the data were normally distributed and thus we determined statistical significance by performing a one-way ANOVA with a post hoc Tukey test. For the results in Figure $4 B$, we performed a Kruskal-Wallis ANOVA on Ranks with post hoc Dunn's test.

\section{Results}

To determine the region of KCC2 that is required for isotonic $\mathrm{K}^{+}-\mathrm{Cl}^{-}$cotransport, we transfected cultured hippocampal neurons with cDNA constructs and, using electrophysiology, assayed their ability to regulate neuronal $\mathrm{Cl}^{-}$. Specifically, gramicidin perforated patch-clamp recordings were used to measure the reversal potential for $\mathrm{Cl}^{-}\left(E_{\mathrm{Cl}}\right)$ from neurons expressing the constructs of interest (as identified by GFP fluorescence; Fig. $1 A$ ). We first transfected neurons with full-length KCC2. As predicted, increased KCC2 expression increased $\mathrm{Cl}^{-}$extrusion, which significantly hyperpolarized $E_{\mathrm{Cl}}(-97.56 \pm 4.49 \mathrm{mV}, n=9, p<$ 0.001 ; Fig. $1 B i, C)$ compared with both vector alone $(-61.46 \pm$ $4.15 \mathrm{mV}, n=6$; Fig. 1 Bii, C) and untransfected neurons in the same culture dish $(-58.44 \pm 2.95 \mathrm{mV}, n=8)$. These data also demonstrate that in our 10-13 DIC neurons, endogenous KCC2 expression is not maximal, as $E_{\mathrm{Cl}}$ still has a range over which it can hyperpolarize. Based on our previous work using a $\mathrm{Rb}^{+}$influx assay that demonstrated that a C-terminal amino acid sequence (amino acids 1022-1037; ISO domain) is required for isotonic constitutive activity of KCC2 in Xenopus oocytes (Mercado et al., 2006), we hypothesized that this same C-terminal domain may confer $\mathrm{KCC} 2$ with the ability to cotransport $\mathrm{K}^{+}$and $\mathrm{Cl}^{-}$in neurons under isotonic conditions. To test this hypothesis, we transfected neurons with a chimeric construct that was generated by replacing the C-terminal amino acids in the ISO domain (amino acids 1022-1037) with the corresponding amino acids from the KCC2 paralog KCC4 (KCC2 $\Delta \mathrm{ISO}$ ); KCC4 is the family member most similar to KCC2, but is not expressed in hippocampal neurons
(Karadsheh et al., 2004). In contrast to KCC2 overexpression, neurons transfected with KCC2 $\Delta \mathrm{ISO}$ did not have significantly hyperpolarized $E_{\mathrm{Cl}}$ values $(-59.19 \pm 11.33 \mathrm{mV}, n=11$; Fig. 1 Bii,C); in fact, the $E_{\mathrm{Cl}}$ of neurons expressing this KCC2 $\Delta \mathrm{ISO}$ construct was not significantly different from neurons expressing the vector alone $(p>$ 0.05). Thus, chimeric KCC2 that lacks the ISO domain is unable to extrude $\mathrm{Cl}^{-}$from neurons under isotonic conditions.

The lack of $\mathrm{KCC} 2$-mediated $\mathrm{Cl}^{-}$-extrusion by KCC2 $\Delta \mathrm{ISO}$ could have resulted from an inability of this chimeric protein to be trafficked to the membrane. To test whether this was the case, we generated extracellular tagged KCC2-HA and KCC2 $2 \mathrm{ISO}-\mathrm{HA}$ constructs ideally suited for quantifying their membrane expression. A triple tandem copy of the influenza virus HA peptide (YPYDVPDYA) was inserted into the second extracellular loop of KCC2 and the tagged, membrane-expressed KCC2 was detected by live immunostaining using an anti-HA antibody. We compared the cluster intensity of anti-HA staining in KCC2 $\Delta$ ISO-HA transfected neurons to KCC2-HA neurons. We found that in neurons transfected with KCC2 $\Delta$ ISO-HA, the normalized cluster intensity was the same as in neurons transfected with KCC2-HA (Fig. $2 A i-i i i, B i ; p=0.5$ ). Both untransfected and transfected neurons incubated with the secondary antibody alone showed no significant $\mathrm{Cy} 3$ fluorescence (data not shown). We also performed gramicidin perforated-patch recordings of the HA-tagged constructs under isotonic conditions to confirm the HA-tagged constructs are functionally equivalent to the original chimeric constructs used in Figure 1. We found that $E_{\mathrm{Cl}}$ of KCC2-HA was significantly hyperpolarized $(-78.71 \pm$ $5.39 \mathrm{mV}, n=10, p<0.05$; Fig. $2 \mathrm{Bii}$ ), when compared with KCC2 $\Delta \mathrm{ISO}-\mathrm{HA}$ alone $(-53.79 \pm 8.56 \mathrm{mV}, n=5$; Fig. $2 \mathrm{Bii})$. When these immunofluorescence results are taken together with our electrophysiological measurements of $E_{\mathrm{Cl}}$, we are able to conclude that the C-terminal 15 amino acid ISO domain is the critical region required for $\mathrm{KCC} 2$-mediated isotonic $\mathrm{Cl}^{-}$transport in neurons.

In addition to being active under isotonic conditions, KCC2 can also be activated by hypotonic shock, which induces cell 


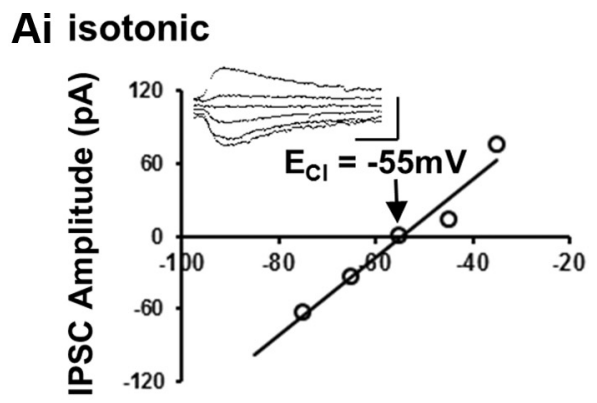

Aii hypotonic

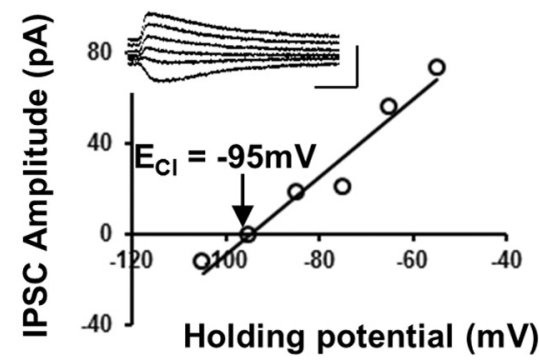

B

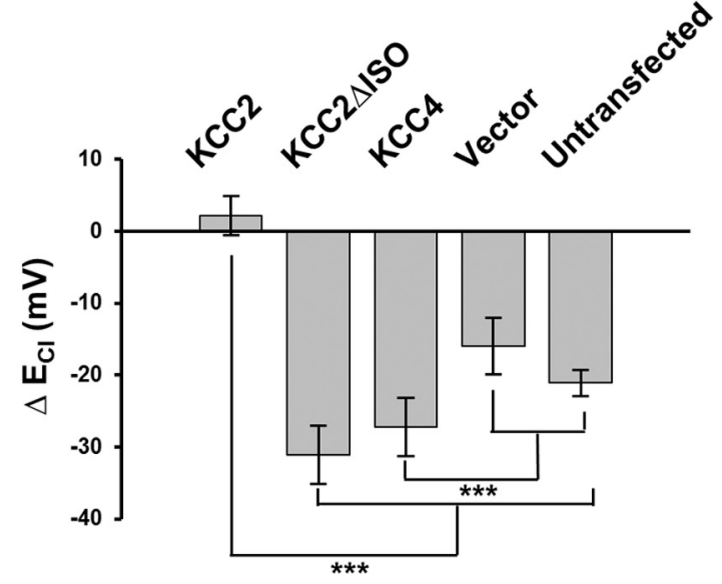

Figure 3. Hypotonic shock recovers $\mathrm{K}^{+}-\mathrm{Cl}^{-}$transport function in $\mathrm{KCC} 2 \Delta \mathrm{ISO}$. $\boldsymbol{A}$, Examples of gramicidin perforated patch-clamp recordings obtained from neurons in hippocampal culture overexpressing KCC2 $\Delta \mathrm{ISO}$ in isotonic ( $300 \mathrm{mOsM}$ ) (i) and hypotonic ( $180 \mathrm{mOsM}$ ) (ii) extracellular solution. The IPSC amplitude was plotted against the holding potential, and the intercept of this curve with the $x$-axis was taken as $E_{\mathrm{Cl}}$ (arrows). Insets, Traces of IPSCs for the examples shown. Scale bars: $100 \mathrm{pA}, 150 \mathrm{~ms}$. $\boldsymbol{B}$, Summary of the change in $E_{\mathrm{c}}$ from isotonic to hypotonic conditions for both untransfected and transfected cultured hippocampal neurons. Transfected neurons overexpressed KCC2, KCC2 $\Delta$ ISO, KCC4, or an empty vector. Data were not paired, but were obtained from separate populations of neurons (in either isotonic or hypotonic conditions). ${ }^{* * *} p<0.001$.

swelling (Lauf and Bauer, 1987). Several other members of the cation-chloride cotransporter family are swelling- or shrinkageactivated and function in ion regulation and cell volume maintenance (Lauf and Bauer, 1987; Flatman, 2008). To determine whether the ISO domain is also required for swelling-induced activation of $\mathrm{KCC} 2$, we again performed gramicidin perforatedpatch recordings and measured $E_{\mathrm{Cl}}$ from neurons expressing the constructs of interest; we induced cell-swelling by exposing neurons to hypotonic conditions (180 mOsM extracellular solution). When we recorded from neurons transfected with KCC2 $\Delta \mathrm{ISO}$, we found that hypotonic shock induced a significant hyperpolarization of $E_{\mathrm{Cl}}$ compared with isotonic conditions $\left[\Delta E_{\mathrm{Cl}}=\right.$ $-31.08 \pm 4.09 \mathrm{mV}$ (from -57.53 to $-88.61 \mathrm{mV}$ ), $n=6, p<$ 0.01 ; Fig. 3]. Hypotonic shock produced no significant change in $E_{\mathrm{Cl}}$ from neurons overexpressing KCC2 (from -97.56 to -95.44
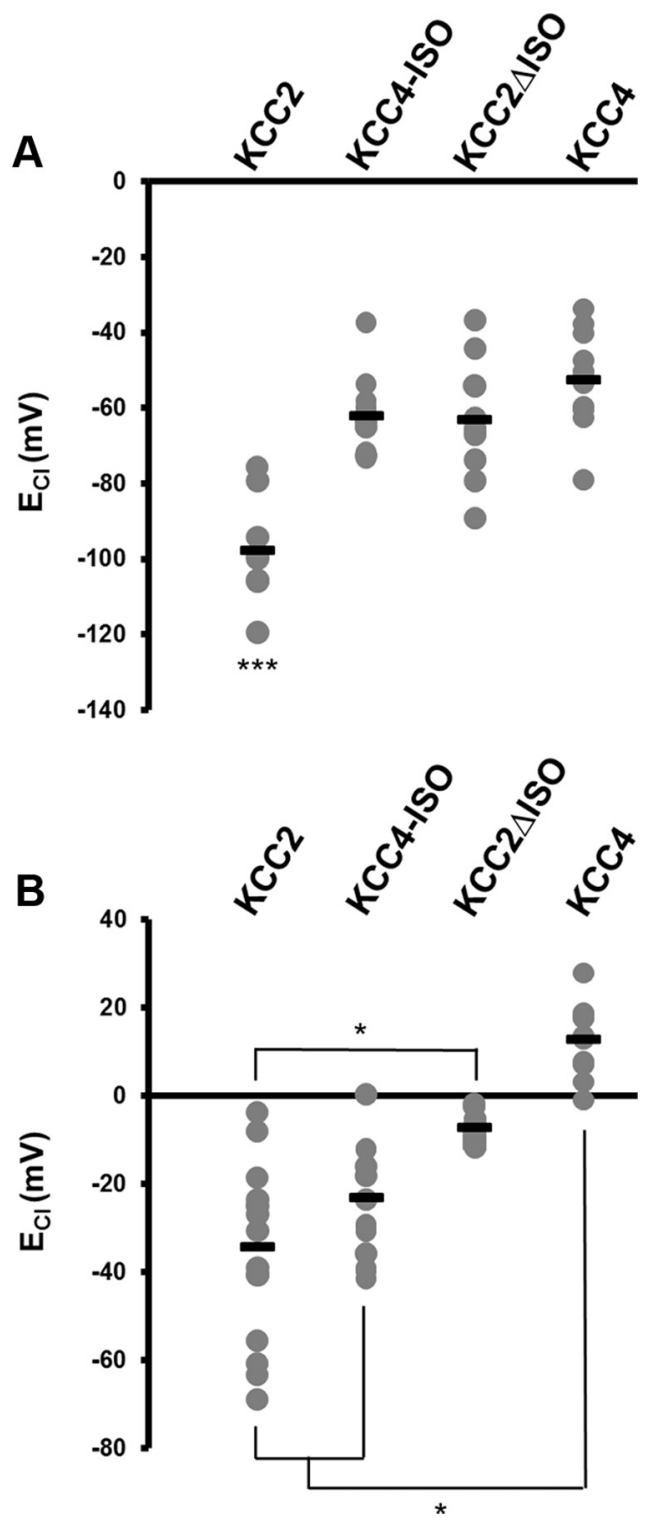

Figure 4. The $K C C 4-I S O$ rescue of isotonic transport depends on neuronal $\mathrm{Cl}^{-} . A$, Summary figure plotting the distribution of $E_{\mathrm{Cl}}$ in neurons overexpressing $\mathrm{KCC}-\mathrm{KCC} 4$ chimeric proteins under isotonic extracellular conditions. $\boldsymbol{B}$, Summary figure plotting the distribution of $E_{\mathrm{Cl}}$ in neurons overexpressing KCC2-KCC4 chimeric proteins and loaded with $50 \mathrm{~mm}$ intracellular $\mathrm{Cl}^{-}$ under isotonic extracellular conditions. ${ }^{*} p<0.05,{ }^{* * *} p<0.001$.

$\mathrm{mV}$ ), because neuronal $\mathrm{Cl}^{-}$was already at a very low concentration ( $4.04 \mathrm{~mm}$, as predicted from the Nernst equation; see Materials and Methods, above). This result is supported by our findings that both untransfected neurons and neurons transfected with an empty vector were significantly hyperpolarized by hypotonic extracellular solution [untransfected: $\Delta E_{\mathrm{Cl}}=$ $-21.05 \pm 1.80 \mathrm{mV}$ (from -58.44 to $-79.5 \mathrm{mV}$ ), $n=9, p<0.01$; transfected: $\Delta E_{\mathrm{Cl}}=-15.97 \pm 3.89 \mathrm{mV}$ (from -61.45 to -77.42 $\mathrm{mV}), n=9, p<0.01$; Fig. $3 B]$. We next compared the swellinginduced activation of KCC2 $\Delta \mathrm{ISO}$ to that of a related family member, KCC4, which is exclusively swelling-activated and not endogenously found in hippocampal neurons (Lauf and Bauer, 1987; Karadsheh et al., 2004). Recordings from KCC4transfected neurons revealed that hypotonic shock induced a similar hyperpolarization of $E_{\mathrm{Cl}}\left(\Delta E_{\mathrm{Cl}}=27.22 \pm 4.04 \mathrm{mV}\right.$ (from -52.31 to $-79.53 \mathrm{mV}), n=11, p<0.01$; Fig. $3 B$ ) when com- 
pared with KCC2 $\Delta \mathrm{ISO}$. Thus, by removing the ISO domain, but maintaining its swelling-activated transport, we have voided KCC2 of its primary neuron-specific function, which demonstrates that there are exclusive molecular determinants of isotonic- and swelling-induced $\mathrm{K}^{+}-\mathrm{Cl}^{-}$cotransport.

In Xenopus oocytes, the KCC2-ISO domain is both required and sufficient for constitutive transport activity, as demonstrated by using a Rb ${ }^{+}$influx assay (Mercado et al., 2006). We next asked whether the ISO domain was also sufficient for constitutive function, by attempting to confer KCC4 with the ability to extrude $\mathrm{Cl}^{-}$from neurons under isotonic conditions. We transfected cultured hippocampal neurons with a KCC2-KCC4 chimeric construct, where we introduced the KCC2 ISO domain into KCC4 (KCC4-ISO). When we made gramicidin perforated patch-clamp recordings from neurons transfected with KCC4-ISO, we found that isotonic $\mathrm{Cl}^{-}$ transport was not conferred $\left(E_{\mathrm{Cl}}=-61.92 \pm 2.83 \mathrm{mV}, n=12\right.$; Fig. $4 A)$ compared with neurons transfected with $\mathrm{KCC} 2\left(E_{\mathrm{Cl}}=\right.$ $-97.56 \pm 4.49 \mathrm{mV}, n=09, p>0.001)$. However, it is known that $\mathrm{K}^{+}-\mathrm{Cl}^{-}$cotransporter activity (including $\mathrm{KCC} 4$ ) is increased at higher levels of intracellular $\mathrm{Cl}^{-}$(Gillen and Forbush, 1999; Bergeron et al., 2006, 2009); thus, we asked whether increased levels of neuronal $\mathrm{Cl}^{-}$were required to reveal whether the addition of the ISO domain to KCC4 could confer this cotransporter with constitutive isotonic activity. We performed this experiment by loading neurons with $50 \mathrm{~mm}$ intracellular $\mathrm{Cl}^{-}$, and found that the ISO domain could in fact confer KCC4 with constitutive isotonic activity. Under these conditions of elevated neuronal $\mathrm{Cl}^{-}$, we found that $\mathrm{KCC} 4$ containing the ISO domain (KCC4-ISO) significantly hyperpolarized these neurons when compared with full-length KCC4 (KCC4ISO: $E_{\mathrm{Cl}}=-23.08 \pm 3.96 \mathrm{mV}, n=14 ; \mathrm{KCC} 4: E_{\mathrm{Cl}}=13.04 \pm 2.49$ $\mathrm{mV}, n=11 ; p<0.05$; Fig. $4 B$ ). Moreover, the $E_{\mathrm{Cl}}$ of neurons overexpressing KCC4-ISO was not significantly different from neurons overexpressing KCC2 (KCC2: $E_{\mathrm{Cl}}=-34.17 \pm 4.93 \mathrm{mV}, n=16 ; p>$ 0.05 ; Fig. $4 B$ ), indicating that the addition of the KCC2 ISO domain to KCC4 can confer KCC4 with constitutive isotonic transport when neuronal $\mathrm{Cl}^{-}$levels have been elevated to promote KCC4 function.

\section{Discussion}

We have discovered the essential C-terminal domain that is required for KCC2 activity in neurons under normal physiological conditions. This C-terminal domain is a 15 aa sequence termed the ISO domain, so named for its ability to confer isotonic transport. By overexpressing KCC2 $\Delta \mathrm{ISO}$, which removes the ability of this transporter to extrude $\mathrm{Cl}^{-}$under isotonic conditions, we have demonstrated a molecular determinant that makes KCC2 unique from its family members. When the ISO domain is replaced with the analogous region in KCC4, the chimeric protein is devoid of transport until activated by cell swelling. Finally, we demonstrate that in addition to being necessary for isotonic transport, the KCC2 ISO domain is also sufficient to drive transport when the ISO domain is inserted into KCC4 under conditions of elevated neuronal $\mathrm{Cl}^{-}$.

All members of the $\mathrm{K}^{+}-\mathrm{Cl}^{-}$cotransporter gene family (Slc12a) are activated by cell swelling, which indicates that there is a commonality between them that mediates this response (Lauf and Bauer, 1987; Kahle et al., 2008). KCC2 is the only family member with the ability to transport $\mathrm{Cl}^{-}$out of neurons under isotonic conditions, suggesting a novel mechanism for KCC2 function. KCC2 does have a unique structural domain, which is comprised of $\sim 100$ aa rich in prolines, serines, and charged residues near the end of the $C$ terminus (amino acids 929-1043); Mercado et al. (2006) initially predicted that this domain may be responsible for isotonic transport in neurons. Inhibiting serine- threonine kinases prevents swelling-induced activation of KCC2 in Xenopus oocytes, but does not impede isotonic transport (Mercado et al., 2006). The precise molecular determinants within the ISO domain that confer neuronal KCC2 with isotonic activity remain to be determined.

When the ISO domain of KCC2 was inserted into KCC4, we were able to restore isotonic $\mathrm{Cl}^{-}$transport when we elevated intracellular $\mathrm{Cl}^{-}$, but not when neuronal $\mathrm{Cl}^{-}$levels were closer to single-digit concentrations that are more routinely observed in mature CNS neurons. Increased intracellular $\mathrm{Cl}^{-}$was likely required to demonstrate the sufficiency of the ISO domain for conferring KCC4 with isotonic transport because $\mathrm{K}^{+}-\mathrm{Cl}^{-}$ cotransporter activity (including KCC4) is increased at higher $\left[\mathrm{Cl}^{-}\right]_{\mathrm{i}}$ (Gillen and Forbush, 1999; Bergeron et al., 2006, 2009). This finding demonstrating the sufficiency of the ISO domain is supported by results obtained in Xenopus oocytes where the same chimeric construct (KCC4-ISO) was sufficient to mediate constitutive activity of the transport. It is well known that KCC2 function is dependent on both phosphorylation state (Strange et al., 2000; Lee et al., 2007; Wake et al., 2007; Rinehart et al., 2009; Watanabe et al., 2009) and oligomerization (Blaesse et al., 2006; Uvarov et al., 2009; Watanabe et al., 2009); it will be interesting in the future to examine whether theses posttranslational modifications regulate transporter activity through an interaction with the ISO domain.

In summary, we have identified that the unique $\mathrm{C}$-terminal ISO domain is required for isotonic KCC2-mediated $\mathrm{Cl}^{-}$extrusion. Furthermore, we demonstrate that there are separate molecular determinants of swelling-activated and isotonic KCC2 cotransport in neurons. The identification of the essential ISO domain in neurons is fundamental for two primary reasons. First, because this domain defines what confers KCC2 with its unique ability to extrude $\mathrm{Cl}^{-}$from neurons at the structural level, allowing this cotransporter to perform its essential physiological role in neuronal $\mathrm{Cl}^{-}$regulation. Second, by identifying the domain required for isotonic KCC2 function, we have revealed a previously unknown target for the development of prevention strategies and therapeutic treatments directed toward neurological disorders associated with disrupted neuronal $\mathrm{Cl}^{-}$regulation and the resulting loss of inhibitory tone.

\section{References}

Balena T, Acton BA, Woodin MA (2010) GABAergic synaptic transmission regulates calcium influx during spike-timing dependent plasticity. Front Synaptic Neurosci 2:16.

Bergeron MJ, Gagnon E, Caron L, Isenring P (2006) Identification of key functional domains in the $\mathrm{C}$ terminus of the $\mathrm{K}+-\mathrm{Cl}-$ cotransporters. J Biol Chem 281:15959-15969.

Bergeron MJ, Frenette-Cotton R, Carpentier GA, Simard MG, Caron L, Isenring $\mathrm{P}$ (2009) Phosphoregulation of $\mathrm{K}(+)-\mathrm{Cl}(-)$ cotransporter 4 during changes in intracellular $\mathrm{Cl}(-)$ and cell volume. J Cell Physiol 219:787-796.

Blaesse P, Guillemin I, Schindler J, Schweizer M, Delpire E, Khiroug L, Friauf E, Nothwang HG (2006) Oligomerization of KCC2 correlates with development of inhibitory neurotransmission. J Neurosci 26:10407-10419.

Blaesse P, Airaksinen MS, Rivera C, Kaila K (2009) Cation-chloride cotransporters and neuronal function. Neuron 61:820-838.

Flatman PW (2008) Cotransporters, WNKs and hypertension: an update. Curr Opin Nephrol Hypertens 17:186-192.

Gillen CM, Forbush B 3rd (1999) Functional interaction of the K-Cl cotransporter (KCC1) with the Na-K-Cl cotransporter in HEK-293 cells. Am J Physiol 276:C328-C336.

Kahle KT, Staley KJ, Nahed BV, Gamba G, Hebert SC, Lifton RP, Mount DB (2008) Roles of the cation-chloride cotransporters in neurological disease. Nat Clin Pract Neurol 4:490-503.

Karadsheh MF, Byun N, Mount DB, Delpire E (2004) Localization of the 
KCC4 potassium-chloride cotransporter in the nervous system. Neuroscience 123:381-391.

Lauf PK, Bauer J (1987) Direct evidence for chloride-dependent volume reduction in macrocytic sheep reticulocytes. Biochem Biophys Res Commun 144:849-855.

Lee H, Chen CX, Liu YJ, Aizenman E, Kandler K (2005) KCC2 expression in immature rat cortical neurons is sufficient to switch the polarity of GABA responses. Eur J Neurosci 21:2593-2599.

Lee HH, Walker JA, Williams JR, Goodier RJ, Payne JA, Moss SJ (2007) Direct protein kinase C-dependent phosphorylation regulates the cell surface stability and activity of the potassium chloride cotransporter KCC2. J Biol Chem 282:29777-29784.

Mercado A, Broumand V, Zandi-Nejad K, Enck AH, Mount DB (2006) A C-terminal domain in $\mathrm{KCC} 2$ confers constitutive $\mathrm{K}+-\mathrm{Cl}-$ cotransport. J Biol Chem 281:1016-1026.

Ormond J, Woodin MA (2009) Disinhibition mediates a form of hippocampal long-term potentiation in area CA1. PLoS One 4:e7224.

Payne JA, Stevenson TJ, Donaldson LF (1996) Molecular characterization of a putative $\mathrm{K}-\mathrm{Cl}$ cotransporter in rat brain. A neuronal-specific isoform. J Biol Chem 271:16245-16252.

Rinehart J, Maksimova YD, Tanis JE, Stone KL, Hodson CA, Zhang J, Risinger M, Pan W, Wu D, Colangelo CM, Forbush B, Joiner CH, Gulcicek EE, Gallagher PG, Lifton RP (2009) Sites of regulated phosphorylation that control K-Cl cotransporter activity. Cell 138:525-536.
Rivera C, Voipio J, Payne JA, Ruusuvuori E, Lahtinen H, Lamsa K, Pirvola U, Saarma M, Kaila K (1999) The K+/Cl- co-transporter KCC2 renders GABA hyperpolarizing during neuronal maturation. Nature 397:251-255.

Strange K, Singer TD, Morrison R, Delpire E (2000) Dependence of KCC2 $\mathrm{K}-\mathrm{Cl}$ cotransporter activity on a conserved carboxy terminus tyrosine residue. Am J Physiol Cell Physiol 279:C860-C867.

Uvarov P, Ludwig A, Markkanen M, Soni S, Hübner CA, Rivera C, Airaksinen MS (2009) Coexpression and heteromerization of two neuronal K-Cl cotransporter isoforms in neonatal brain. J Biol Chem 284:13696-13704.

Wake H, Watanabe M, Moorhouse AJ, Kanematsu T, Horibe S, Matsukawa N, Asai K, Ojika K, Hirata M, Nabekura J (2007) Early changes in KCC2 phosphorylation in response to neuronal stress result in functional downregulation. J Neurosci 27:1642-1650.

Watanabe M, Wake H, Moorhouse AJ, Nabekura J (2009) Clustering of neuronal $\mathrm{K}+-\mathrm{Cl}-$ cotransporters in lipid rafts by tyrosine phosphorylation. J Biol Chem 284:27980-27988.

Woodin MA, Ganguly K, Poo MM (2003) Coincident pre- and postsynaptic activity modifies GABAergic synapses by postsynaptic changes in $\mathrm{Cl}-$ transporter activity. Neuron 39:807-820.

Zhao B, Wong AY, Murshid A, Bowie D, Presley JF, Bedford FK (2008) Identification of a novel dileucine motif mediating $\mathrm{K}(+) / \mathrm{Cl}(-)$ cotransporter KCC2 constitutive endocytosis. Cell Signal 20:1769-1779. 